\title{
Ärzte können auf Nachschlag hoffen
}

\section{Ohrfeige für die KV Sachen: Deren Honorarverteilung zwischen 2005 und 2008 entsprach nicht den Gesetzes-Vorgaben, urteilte das Bundessozialgericht. Da- durch könnten hunderte Ärzte einen Nachschlag bekommen - und vier weitere KVen müssen ihre Regelungen offenbar überdenken.}

Die Honorarverteilung der KV Sachsen von 2005 bis 2008 war rechtswidrig. Sie entsprach nicht den gesetzlichen Vorgaben für die Regelleistungsvolumina (RLV), urteilte am 9. Mai das Bundessozialgericht (BSG) in Kassel.

\section{Nur Hessen und Niedersachsen setzten Vorgaben um}

Vermutlich mehrere Hundert Ärzte, die Widersprüche eingelegt haben, können auf einen Nachschlag hoffen. Der Bewertungsausschuss wollte die RLV eigentlich schon weitgehend flächendeckend 2005 zum neuen Einheitlichen Bewertungsmaßstab (EBM) einführen. Allerdings gab es eine Öffnungsklausel. Danach durften die KVen statt der RLV alte Regelungsmechanismen fortführen, wenn diese eine vergleichbare Mengensteuerung bewirken. Danach hatten sich nur Hessen und Niedersachsen auf die RLV eingelassen und die Vorgaben des Ausschusses weitgehend umgesetzt. In zahlreichen anderen KVen ist seitdem streitig, ob die fortgeführten Regelungen den Vorgaben noch entsprechen. Im März 2010 hatte das BSG bereits die Honorar- verteilung in Baden-Württemberg und im Dezember 2011 die der KV Nordrhein verworfen: Baden-Württemberg habe keinen einheitlichen Punktwert eingeführt, Nordrhein keine Arztgruppenbezogenen Mengenbegrenzungen.

Sachsen hatte mit arztgruppenspezifischen Anpassungsfaktoren die Individualbudgets auf einen einheitlichen Punktwert von 3,75 Cent umgerechnet. Im Ergebnis blieben aber trotzdem praxisbezogene Mengenbegrenzungen erhalten. Damit habe auch Sachsen die Vorgaben nicht ausreichend umgesetzt, urteilte nun das BSG. Die Öffnungsklausel habe nur die Fortführung von Altregelungen mit den RLV vergleichbarer Wirkung erlaubt. Ziel des Gesetzgebers sei dabei eine "durchschnittsorientierte Nivellierung“ gewesen. Diese habe es in Sachsen trotz des Anpassungsfaktors nicht gegeben. Die Regelung habe weiterhin große Praxen begünstigt.

\section{Mehrere Hundert betroffen}

Damit gab das BSG einer HNO-Ärztin und einer Neurologin weitgehend recht. Nach Angaben der KV haben überwie- gend HNO-Ärzte Widerspruch gegen ihre Honorarbescheide eingelegt.

Rechtsanwalt Jan Immen, der vor dem BSG die Ärztinnen vertreten hat, schätzt die Zahl der Betroffenen in Sachsen auf insgesamt mehrere Hundert. Soweit sich die Ärztinnen auch gegen die Vergütung der Neurologen sowie die Begrenzung der Arztzahlen richteten, wies das BSG ihre Klagen aber ab. Nach dem Kasseler Urteil müssen wohl auch weitere KVen ihre damaligen Regelungen überdenken, etwa Schleswig-Holstein, Berlin, Bremen und eventuell auch Bayern. Dagegen sagte ein Vertreter der KV Hamburg, die Hamburger Regelungen seien mit dem Sachsen-Urteil nicht vom Tisch; die KV werde sie daher vom BSG prüfen lassen.

$(m w)$

www.aerztezeitung.de lungsverhältnis zurückgedrängt. Der Patient darf frei wählen, von wem er sich behandeln lassen möchte und der Arzt kann sich im Rahmen des Behandlungsverhältnisses auf die Interessen des Patienten konzentrieren.

\section{Kein Freibrief für Vorteilsgewährung}

Um Missverständnissen vorzubeugen: Selbstverständlich ist die Entscheidung kein Freibrief für die Vorteilsgewährung zwischen Pharmaindustrie und Vertragsärzten. Berufsrechtlich sind Verordnungen gegen Entgelt - wie immer dieses ausgestaltet ist - weiterhin untersagt.

Die Unabhängigkeit der ärztlichen Entscheidung ist wesentlicher Bestandteil des ärztlichen Berufsbildes. Vertragsarzt- rechtlich setzt das Gebot der Wirtschaftlichkeit der Versorgung Grenzen und die Forderung oder Annahme unzulässiger Zuwendungen für die Arzneimittelverordnung verstößt nach den Neuregelungen des GKV-VStG sogar explizit gegen vertragsärztliche Pflichten. Die Vorteilsgewährung von Pharma-Unternehmen bleibt daher ebenso wie vergleichbare Absprachen mit Laboren, Kollegen, Apotheken o.ä. als unerlaubte Zuwendungen oder unerlaubte Zuweisungen unzulässig und kann disziplinarrechtliche und wettbewerbsrechtliche Konsequenzen haben, ungeachtet der steuerlichen Problemstellungen. Nur in engen Grenzen und dann, wenn eine werthaltige Gegenleistung durch den Arzt erbracht wird und die
Vergütung angemessen ist, wie etwa bei Studien und Vortragstätigkeiten, ist die Annahme von Honoraren rechtlich nicht zu beanstanden.

Primäre Verantwortung bleibt Patient Zum Beauftragten der Krankenkassen mutiert der Vertragsarzt durch diese Regelungen jedoch nicht. Der Bundesgerichtshof hat deutliche Worte gesprochen, wenn er betont, dass der Vertragsarzt neben der Verpflichtung zur Beachtung berufsrechtlicher und anderer Grundsätze gegenüber Krankenkassen und Kassenärztlichen Vereinigungen, auch bei der Verordnung von Arznei-, Heil- und Hilfsmitteln primär seinem $\mathrm{Pa}$ tienten gegenüber verantwortlich bleibt. 